\title{
A Local Linear Modeling Paradigm with a Modified Counterpropagation Network
}

\author{
Jeongho Cho, Jose C. Principe and *Mark A. Motter \\ Computational NeuroEngineering Lab., University of Florida, Gainesville, FL 32611 \\ *Dynamics and Control Branch, NASA Langley Research Center, Hampton, VA 23681
}

\begin{abstract}
The Counter-Propagation neural network (CPN) was selected to investigate the modeling problems because it integrates both supervised and unsupervised learning. The network is taught to have clusters that are described by codebook vectors in the training phase. The basic CPN algorithm is modified incorporating the Local Linear Models (LLMs) to provide functional mappings and identify potentially nonlinear plants. The objective is a reduction of the approximation error in a CPN. In this framework the quantization error in the input space serves as a basis for the LLMs in the output space. The performance of the proposed algorithms is tested on the nonlinear dynamic system and shows the influence of this modification on the system identification quality.
\end{abstract}

\section{INTRODUCTION}

Dynamic system modeling is the discipline interested in building mathematical models of nonlinear systems, starting from experimental time series data, measurements, or observations. Typically, a certain linear or nonlinear model structure which contains unknown parameters is chosen by the user. The resulting model of a system is often very important for analysis, simulation, prediction, monitoring, diagnosis, and control system design [10].

Modeling techniques of the nonlinear dynamic system can generally be classified as global and local models. Global models are constructed once and fit all the state space, while local models represent local regions [12]. More recently, the dynamic neural network paradigm has provided a promising alternative for the processing of nonlinear signals [10]. Although their universal approximation capabilities has been proved, a number of global models such as Multi-Layered Perceptrons (MLPs) or Radial Basis Functions (RBFs) neural networks present some limits for function approximation. Also, global models attempt to yield a compact representation of an underlying dynamical system and require relatively few tunable parameters. On the other hand, local models lack the property of compactness and are not so economical, but have the ability to adhere to the local shape of an arbitrary surface, which is difficult especially in cases when the dynamical system characteristics vary considerably throughout the state space. Therefore, in a number of cases local models outperforms global ones [12][13].
Local Modeling is usually based on nearest-neighbor methods. Once the input vector is available, the nearest neighbors in the data set are located and a simple model is constructed using only the neighboring points. After the model is constructed, it is evaluated using the input vector to produce the local model output [1][5][7][12]. As Farmer and Sidorowich [13] have already shown, local linear models, despite their simplicity, provide an effective and accurate approximation, while high-order polynomials in higher dimensions are not significantly better than the first-order ones.

Building local mappings in all points of reconstructed state space is a time and memory consuming process, which led to a natural idea to quantize the state space and build local mappings only in positions given by obtained prototype vectors. In most of the neural networks papers, the Self-Organizing Map (SOM) has been used to quantize the feature space [5][6]. Principe et al. [1] used the SOM based local linear modeling for the task of prediction of chaotic time series as well as for the design of switching controllers. Recently, it is proposed a scheme that essentially followed local linear modeling based on SOM topology for nonautonomous system [7][8].

Self-Organizing Map (SOM) is a topology that converts complex, nonlinear statistical relationships of highdimensional data into simple geometric relationships and quantize the feature space [2]. The role of the SOM is to divide the input space into a set of regions represented by the weights of each processing element (PE). Each of these PEs is located in the input space. Any point of a cluster is projected onto the closest corresponding PE of the map according to the Euclidean distance. That kind of projection can be useful in classification process but brings about the poor performance of SOM in function approximation because of its discrete representation of the data [7][9].

Thus, we tried to reduce the approximation error with LLMs based on a CPN, which are hybrid networks that combine supervised and unsupervised learning to create a self-organizing look-up table that can be used for function approximation [11]. The advantage of this network over conventional look-up tables is that the Kohonen map provides for a statistically optimal coverage of the input space even if the mathematical form of the underlying function is completely unknown. As input feature vectors from a training set are presented to the network, unsupervised learning is used to create a topologypreserving (Kohonen) map of the input data while, at the 
same time, supervised learning is used to associate an appropriate output feature vector with each PE on the map [11]. Since the output at each PE is just the average output for all of the feature vectors that map to that point LLMs are created for better approximation using the quantization error in the input space and the average output. This is achieved by coupling each PE with a linear mapping in such a way that a functional relationship can be established between each Voronoi region in the input space (of the SOM) and the desired signal. The proposed system identification scheme was tested using a nonlinear dynamic system.

\section{EMBEDDING AND LOCAL LINEAR MODELING}

Neural network based modeling methods had proven successful in modeling autonomous nonlinear dynamical systems [1][4][5][6] to the modeling of nonautonomous dynamical systems [7][8][10]. The temporal state evolution of an autonomous system is functionally dependent only on the system state, but nonautonomous systems allow for an explicit dependence on an independent variable in addition to the system state. This independent variable is taken to be an external, or exogenous driving signal, referred to as the control input. For an autonomous system, it is reasonable to assume that the future behavior, or output, of the system can be predicted over some finite interval from a finite number of observations of past outputs [3]. In contrast, predictions of the behavior of a nonautonomous system require consideration of not only the past outputs in response to past inputs, but the future input to the system as well. In this paper, we consider a nonautonomous system.

In most real-life problems, the dynamic equations describing the system dynamics are unknown. Therefore the task of modeling is to find $\tilde{f}$ as an approximation of the functional model $f$ in $\vec{y}(k+1)=f[\vec{y}(k), \vec{u}(k)]$ to preserve the same dynamical properties from output vector $\vec{y}(k) \in \mathfrak{R}^{p}$ and input vector $\vec{u}(k) \in \mathfrak{R}^{q}$. If no or insufficient physical insight is available, but only observed data (the only information about the process consists in the values of the inputs and the measured outputs), no fixed model structure can be assumed a priori. In this case, one must resort to black-box models which are usually chosen to be input-output models. The input-output modeling approach for neural networks has been partly introduced and illustrated in [10].

Delay-coordinate embedding is one way to help the input-output modeling; it lets one reconstruct the internal dynamics of a complicated nonlinear system from a single time series. That is, one can often use delay-coordinate embedding to infer useful information about internal (and immeasurable) states using only output information. The reconstruction produced by delay-coordinate embedding is not, of course, completely equivalent to the internal dynamics in all situations [4][13]. However, a single-sensor reconstruction, if done right, can still be extremely useful because its results are guaranteed to be topologically (i.e. qualitatively) identical to the internal dynamics. This means that conclusions drawn about the reconstructed dynamics are also true of the internal dynamics of the system inside the black box.

To reconstruct the dynamics, we begin with experimental data which are output vector, $\vec{y}(k)$ and input vector, $\vec{u}(k)$. Delay-coordinate reconstruction of the unobserved and possibly multi-dimensional phase-space dynamics from this vector, $\vec{s}(k)=[\vec{y}(k), \vec{u}(k)] \in \mathfrak{R}^{p+q}$, is governed by two parameters, embedding dimension $d_{E}$ and time delay $\tau$. The resultant model is given by:

$$
\tilde{y}(k+1)=f\left[\vec{s}(k), \cdots, \vec{s}\left(k-\left(d_{E}-1\right) \tau\right]=f(\overrightarrow{\mathbf{s}}(k))\right.
$$

where $\overrightarrow{\boldsymbol{s}}(k) \in \mathfrak{R}^{(p+q) d_{E}}$. Proper choice of $d_{E}$ and $\tau$ is critical to this type of phase-space reconstruction and must therefore be done wisely; only "correct" values of these two parameters yield embeddings that are guaranteed - by the Taken's theorem [3] and subsequent work by Packard et al [14] and Casdagli et al [4] - to be topologically equivalent to the original (unobserved) phase-space dynamics.

Assume that a time varying linear model of a signal generating process is given by

$$
f(\overrightarrow{\boldsymbol{s}}(k))=b(k)+\vec{a}(k)^{T} \overrightarrow{\boldsymbol{s}}(k)
$$

where $\vec{a}(k)=\left[a_{1}(k), \cdots, a_{(p+q) d_{E}}(k)\right]^{T}$ and $b(k) \in \Re$ are a set of unknown time-varying parameters. And the SOM is designed such as its output $\tilde{y}(k+1)$, related to a given input $\overrightarrow{\boldsymbol{s}}(k)$, is provided by a winning PE. Then this model can be identified with the first order Taylor series approximation of the function $f(\overrightarrow{\boldsymbol{s}}(k))$ in the neighborhood of the center point (prototype weight of the winner in the input space) $w_{i^{o}}(k)$ described by the relation:

$$
\begin{aligned}
f(\overrightarrow{\boldsymbol{s}}(k)) & =f\left(\boldsymbol{w}_{i^{o}}^{i n}(k)+\Delta \overrightarrow{\boldsymbol{s}}(k)\right) \\
& \cong f\left(\boldsymbol{w}_{i^{o}}^{i n}(k)\right)+\frac{\partial f\left(\boldsymbol{w}_{i^{o}}^{i n}(k)\right)}{\partial \overrightarrow{\boldsymbol{s}}(k)} \cdot \Delta \overrightarrow{\boldsymbol{s}}(k)+\text { h.o.t }
\end{aligned}
$$

where $\Delta \overrightarrow{\boldsymbol{s}}(k)=\overrightarrow{\boldsymbol{s}}(k)-\boldsymbol{w}_{i^{o}}^{\text {in }}(k)$ and

$$
\frac{\partial f\left(\boldsymbol{w}_{i^{o}}^{i n}(k)\right)}{\partial \overrightarrow{\boldsymbol{s}}(k)}=\left[\frac{\partial f\left(\boldsymbol{w}_{i^{o}}^{i n}(k)\right)}{\partial s_{l}(k)}, \cdots, \frac{\partial f\left(\boldsymbol{w}_{i^{o}}^{i n}(k)\right)}{\partial s_{(p+q) d_{E}}(k)}\right] .
$$

And, since we know the mapping from the center point of input space to the center point of output space (i.e. $\left.\boldsymbol{w}_{i^{o}}^{\text {in }} \rightarrow \boldsymbol{w}_{i^{o}}^{\text {out }}\right)$

$$
f(\overrightarrow{\boldsymbol{s}}(k)) \cong \boldsymbol{w}_{i^{o}}^{\text {out }}(k)+\frac{\partial f\left(\boldsymbol{w}_{i^{o}}^{\text {in }}(k)\right)}{\partial \overrightarrow{\boldsymbol{s}}(k)} \cdot \Delta \overrightarrow{\boldsymbol{s}}(k)+\text { h.o.t }
$$

The higher order terms (h.o.t) can be neglected when the estimation concerns a sufficiently small neighborhood centered on $\boldsymbol{w}_{i^{o}}^{\text {in }}(k)$. For each PE, the first order partial 
derivatives $\partial f\left(\boldsymbol{w}_{i^{o}}^{\text {in }}(k)\right) \partial \overrightarrow{\boldsymbol{s}}(k)$ corresponding to $\vec{a}(k)$ is estimated but $b(k)$ corresponding to the zero order term $\boldsymbol{w}_{i^{o}}^{\text {out }}(k)$ is not, since CPN algorithm creates $\boldsymbol{w}_{i^{o}}^{\text {out }}(k)$.

\section{LOCAL LINEAR MODELING BASED ON A COUNTERPROPAGATION NETWORK}

We utilize a modified CPN (Kohonen learning for both input and output space) to partition the input data to smaller regions by associating input data with their winning PE, constructed through competitive learning such that the trained neural field bears a strong global resemblance to the input space and thus to use as a basis for further processing [8]. Also it is exploited to make use of the average outputs corresponding to partitioned input space. Therefore the CPN is employed as a modeling infrastructure to construct the LLMs. They are created by fitting a model to the data in the Voronoi tessellation. By way of doing this, we can create models that are local to the data in the Voronoi tessellation. Our work shows that the dynamics can be modeled by a set of local models, each directly fitted to the quantized inputoutput joint space obtained from the CPN. The proposed nonlinear modeling scenario follows two steps: training of the $\mathrm{CPN}$ and estimation of the local linear models.

\subsection{Training of a modified $C P N$}

For data-evaluation tasks, an output layer is added to the SOM, which results in a counterpropagation architecture shown in Figure 1 since the original SOM is known to perform poorly on regression problems. Training of the modified CPN is accomplished via the Kohonen learning process [2] so that during the first phase of learning, the input vectors are clustered and during the second phase, the weights from the clustered PEs to the output PEs are adapted to produce the desired response.

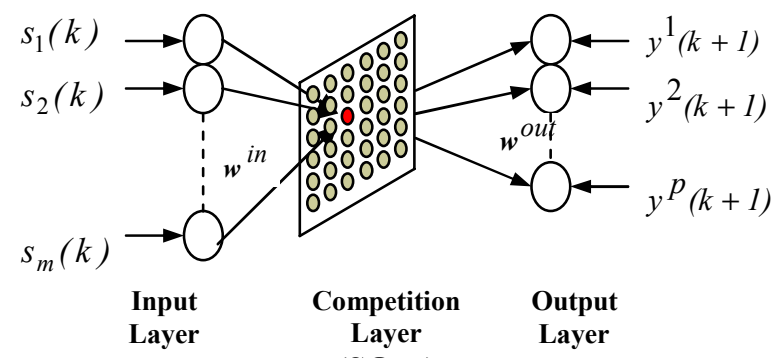

Figure 1. Architecture of the counterpropagation network

Let $\overrightarrow{\boldsymbol{s}}(k) \in \Re^{m}$ denote the input vector for the CPN and $\vec{y}(k+1) \in \Re^{p}$ the desired output vector. And $\boldsymbol{w}_{i}{ }^{\text {in }}, \boldsymbol{w}_{i}{ }^{\text {out }}$ denote the weight vector of PE $i$ of the input layer and the output layer, respectively. With each vector, $\overrightarrow{\boldsymbol{s}}(k)$ which consists of $\vec{u}(k)$ and $\vec{y}(k)$ as well as their delayed samples, presented as the input to the network, the Kohonen learning algorithm adaptively discretizes the continuous input space into a set of $\mathrm{K}$ disjoint Voronoi cells. The response of a CPN to input $\overrightarrow{\boldsymbol{s}}(k)$ is determined by the reference vector $\boldsymbol{w}_{i}{ }^{i n}$ of the $\mathrm{PE}$ that produces the best match to the input.

$$
i^{o}=\arg \min _{i}\left\{\left|\overrightarrow{\boldsymbol{s}}(k)-\boldsymbol{w}_{i}^{i n}(k)\right|\right\}
$$

Then the $k^{\text {th }}$ adaptation of the weights is done in the following manner.

$$
\begin{aligned}
& \boldsymbol{w}_{i}^{\text {in }}(k+1)=\boldsymbol{w}_{i}^{\text {in }}(k)+\eta(k) \Lambda_{i}(k)\left(\overrightarrow{\boldsymbol{s}}(k)-\boldsymbol{w}_{i}^{\text {in }}(k)\right) \\
& \boldsymbol{w}_{i}^{\text {out }}(k+1)=\boldsymbol{w}_{i}^{\text {out }}(k)+\eta(k) \Lambda_{i}(k)\left(\vec{y}(k+1)-\boldsymbol{w}_{i}^{\text {out }}(k)\right)
\end{aligned}, i=i^{o}
$$

where $\eta(k)$ is the learning rate and $\Lambda_{i}(k)$ is a typical neighborhood function. Each new feature vector presented to the network will trigger a response that is the average for those feature vectors closest to it in the input data space.

\subsection{Estimation of the local linear models}

The modified CPN preserves topological relationships in the input space in such a way that neighboring inputs are mapped to neighboring PEs in the map space. Then, when each PE is extended with a linear model it can actually learn the mapping $f$ in a supervised way. Each $\mathrm{PE}$ has an associated linear model $\vec{a}_{i}$ that represents the linear approximation of the local dynamics in the $i^{\text {th }}$ Voronoi cell.

The linear model weights $\vec{a}_{i}$ are computed directly from the desired samples $y_{i}^{l}$ in a cluster and the clustered inputs by a least square fit within a local neighborhood centered at the current winning PE. The size of the neighborhood must be at least equal to the input space dimension. The design procedure for this linear model is as follows:

1) Apply training data to the CPN and find the winning PE corresponding to the inputs such that we have winner-input pairs.

2) Use the least square fit to find the local linear model coefficients for the winning PE, $i^{o}$, where desired outputs $y_{i j}^{l} \in \mathfrak{R}^{\mathcal{N}_{B} \cdot M_{j}}$ within a local neighborhood size of $\mathcal{N}_{B}$ as

$$
y_{i j}^{l}=w_{i^{o} j}^{\text {out }}+\vec{a}_{i^{\circ}}^{T}\left(\overrightarrow{\boldsymbol{s}}_{i j}-\boldsymbol{w}_{i^{o} j}^{i n}\right) \quad \text { for } \forall j \in \mathcal{N}_{B}, \quad \forall i \in M_{j}
$$

where $M_{j}$ is the size of inputs involved in $j^{\text {th }} \mathrm{PE}$, $w_{i^{o} j}{ }^{o u t} \in \mathfrak{R}^{\mathcal{N}_{B}}$ are the prototype output weights from $i^{o}$ to $j^{\text {th }}$ PE and $\overrightarrow{\boldsymbol{s}}_{i j}$ are the input vectors of the winning PE and input vectors of the $j^{\text {th }}$ closest neighbor to the winner.

3) In testing, once the winning PE is determined we select the appropriate linear model from the list of associated models. Apply the local model to obtain the estimated output.

$$
\tilde{y}^{l}(k+1)=w_{i^{o}}^{\text {out }}+\vec{a}_{i^{o}}^{T}\left[\overrightarrow{\boldsymbol{s}}(k)-\boldsymbol{w}_{i^{o}}^{\text {in }}\right]
$$




\section{SIMULATION RESULTS}

In this section, we present some simulation results obtained from the application of the proposed method on the nonlinear system chosen from [10] and has the form

$$
y(k+1)=\frac{y(k)}{1+y(k)^{2}}+u(k)^{3}
$$

As we can see in the above equation, it is very difficult to model the above nonlinear system with multiple linear models because of the terms, $y(k)^{2}$ and $u(k)^{3}$, if we select the linear model as $y(k+1)=a_{1} y(k)+a_{2} y(k-1)+\cdots+$

$b_{1} u(k)+b_{2} u(k-1)+\cdots$. However, since we identify the system utilizing linear models based on the quantization error in the input Voronoi tessellation we can resolve this difficulty.

A total of 100 PEs, arranged on a $10 \times 10$ square lattice, constitute the CPN neural field of the input space. We selected an embedding dimension of $d_{E}=2$ and $\tau=1$ for output $y(k)$ and control input $u(k)$. Thus the number of input parameters is 4 . The CPN is trained with 2000 samples for 8000 epochs. The training samples were obtained by exciting the system using the random control inputs in the interval [-1.5 1.5]. After quantization of the input-output joint space, LLMs were built.

The trained model was tested on a new random sequence of 500 samples. Signal-to-Error Ratio (SER) of testing data with LLMs based on CPN was around $29.22 \mathrm{~dB}$ while without LLMs we obtained SER of about $19.44 \mathrm{~dB}$. The actual and identified trajectory of the system and the corresponding errors using CPN and the proposed algorithm (i.e. CPN+LLMs), are presented in Figure 2 and Figure 3, respectively. Figure 3 shows clearly the benefit of the LLMs. The estimated output was pretty close to the actual output except at those points where there is a sudden discontinuity while the standard CPN estimation in Figure 2 clearly deviates from the desired signal.
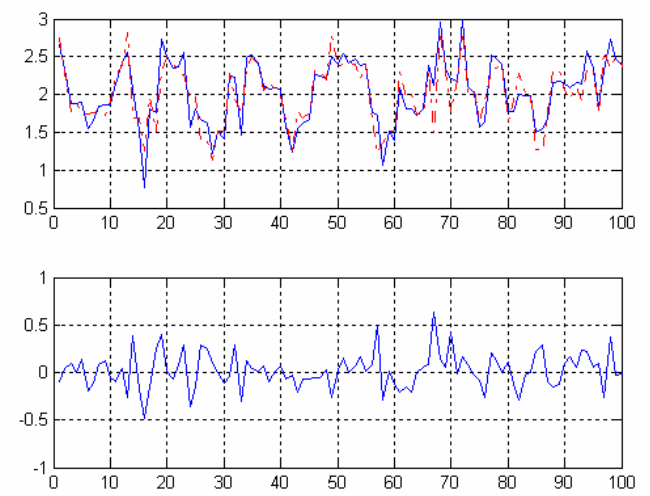

Figure 2. The estimate from the CPN vs. the desired output and corresponding error.
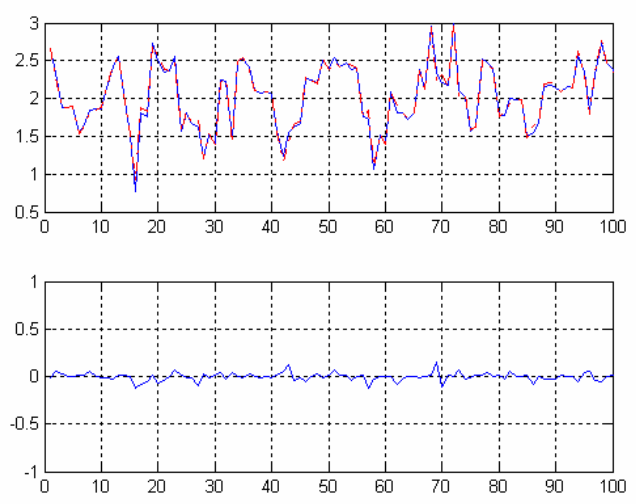

Figure3. The estimate from LLMs based on CPN vs. the desired output and corresponding error.

\section{CONCLUSION}

There has been an increased interest on LLMs for numerous engineering applications. In this paper, we have combined CPN, which is based on a combination of clustering of input and averaging of output for all of the feature vectors, with the simplicity of local linear modeling for function approximation. The proposed architecture involves a CPN that determines the region where the state dynamics of the observed system is and selects an average output of the region as well as an appropriate local linear model which is created by the quantization error in the input space. The selected linear model, which is optimized to represent the local dynamics, is then used to produce an estimate of the system output.

In simulations, we have verified that the CPN successfully quantizes the input-output joint space and, based on this, the linear models produce promising results.

\section{ACKNOWLEDGEMENTS}

This work was partially supported by Accurate Automation Corporation under grant \#463, and NASA grant NAG-102068 .

\section{REFERENCES}

[1] J.C. Principe, L. Wang and M.A. Motter, "Local Dynamic Modeling with Self-Organizing Maps and Applications to Nonlinear System Identification and Control," Proc. of IEEE, Vol. 86, No. 11, pp.22402258, 1998.

[2] Kohonen, T., Self-Organizing Maps, Springer, 1995.

[3] F. Takens, "Detecting strange attractors in turbulence," in Dynamical Systems and Turbulence (Springer Lecture Notes in Mathematics), Vol. 898, pp. 365-381, 1980. 
[4] M. Casdagli, "Nonlinear prediction of chaotic time series," Phys. D, 35(3), pp.335-356, 1989.

[5] J. Vesanto, "Using the SOM and Local Models in Time-Series Prediction," Proc. WSOM, pp.209-214, 1997.

[6] J. Walter, H. Ritter and K. Schulten, "Non-linear Prediction with Self-organizing Maps," Proc. IJCNN, Vol. 3, pp.589-594, 1990.

[7] D. Moshou and H. Ramon, "Extended Self-Organizing Maps with Local Linear Mappings for Function Approximation and System Identification," Proc. WSOM, pp.181-186, 1997.

[8] J. Göppert and W. Rosenstiel, "Varying Cooperation in SOM for Improved Function Approximation," Proc. ICNN, Vol. 1, pp.1-6, 1996.

[9] M. Aupetit, P. Massotte and P. Couturier, "C-SOM: A Continuous Self-Organizing Map for Function Approximation," Proc. Intelligent System and Control, 1999.

[10] K. Narendra and K. Parthasarathy, "Identification and Control of Dynamical Systems using Neural Networks," IEEE Trans. Neural Networks, Vol.1, No.1, pp.4-27, 1990.

[11] Hecht-Nielsen, R., Neurocomputing, Addison-Wesley, 1990.

[12] A.C. Singer, G.W. Wornell and A.V. Oppenheim, "Codebook Prediction: A Nonlinear Signal Modeling Paradigm," IEEE ICASSP, pp.325-328, 1992.

[13] J.D. Farmer and J.J. Sidorowich, "Predicting Chaotic Time Series," Physical Review Letters, 59(8), pp.845848, 1987.

[14] N.H. Packard, J.P. Crutchfield, J. D. Farmer, and R. S. Shaw, "Geometry from a time series," Phys. Rev. Let., 045:712, 1980. 